\title{
Author's reply to comments on "Continuity of care between hospital pharmacies and community pharmacies, and costs avoided: a pilot experience in times of COVID-19 in Spain"
}

Dear Editor,

We would like to thank the Pharmacy Department of the Marques de Valdecilla University Hospital (HUMV) for their comments and clarifications regarding our article titled "Continuity of care between hospital pharmacies and community pharmacies, and costs avoided: a pilot experience in times of COVID-19 in Spain."

We are glad to know that our attempt to quantify the costs derived from the experience carried out last year in Cantabria has reached acknowledgment and interest for clarification. It was a pilot experience aimed to relieve the hospital from the increased burden caused by the COVID-19 pandemic and to spare patients unnecessary risks at a time of exceptional sanitary and social crisis. In fact, this was not the only Spanish region to develop such an approach, benefiting from telepharmacy.

As we stated in the article, the hospital pharmacy of HUMV was still responsible for dispensing the drugs, while informed deliveries of such drugs were carried out by community pharmacists. Bidirectional communication between the hospital pharmacy and the community pharmacy was prompted to avoid problems related to patient delivery data, as well as discrepancies between documentation and dispensed medication. In contrast with the use of a private courier, this channel of communication allowed drugs to be delivered within the pharmaceutical channel throughout the whole process.

Regarding the social costs derived from the initiative, on the one hand, we acknowledge that a relevant percentage of the patients who participated were not working at that time. Therefore, the real productivity losses averted could have been lower than estimated. Moreover, as in the majority of health-economic evaluations, we assessed productivity losses using the human capital approach that considers the whole professional life and therefore long-term consequences. A friction cost approach that assumes that vacancies can be covered with previously unemployed or already employed workers would have undoubtedly reduced our estimations. On the other hand, caregiver productivity losses were not estimated, nor intangible costs monetized (i.e., patient suffering prevented thanks to the initiative). Intangible costs are difficult to measure and monetize. Though usually omitted, these should not be forgotten, especially during this exceptional situation where patients and their families would probably be willing to pay to avoid hospital visits.

Also, as in every economic estimation, certain assumptions were made as we stated in our limitations (e.g., average disposable income adjusted by the population size of the municipality).

Nevertheless, the relevant aspect is not the exact value of avoided cost, but the acknowledgment that certain social costs exist and have to be considered in the initiative as a whole. We want to highlight the importance of management innovations such as this initiative, led by extraordinary circumstances. We already stated that a strategy with shipment costs entirely borne by community pharmacies and/or distribution warehouses is not sustainable over time. Also, to be efficient, this type of service should not imply an extra cost over existing alternatives for the healthcare system.

This exceptional situation may be a good opportunity to improve the coordination between hospital pharmacies and community pharmacies in Spain, in order to bring benefits not only for the patients but also for the health system and the society as a whole. Further research is encouraged to enhance the available evidence about the impact of an improved coordination on the health outcomes.

Received: April 21, 2021

Accepted: April 25, 2021

Published online: May 4, 2021

Corresponding author:

Néboa Zozaya

Calle Moreto, 17

$5^{\circ}$ Dcha. 28014

Madrid - Spain

neboa.zozaya@weber.org.es 